\title{
First AU-scale observations of V1647 Orionis with VLTI/MIDI*
}

\author{
P. Ábrahám ${ }^{1}$, L. Mosoni ${ }^{1}$, Th. Henning ${ }^{2}$, Á. Kóspál ${ }^{1}$, Ch. Leinert ${ }^{2}$, S. P. Quanz ${ }^{2}$, and Th. Ratzka ${ }^{2}$ \\ 1 Konkoly Observatory of the Hungarian Academy of Sciences, PO Box 67, 1525 Budapest, Hungary \\ e-mail: abraham@konkoly.hu \\ 2 Max-Planck-Institut für Astronomie, Königstuhl 17, 69117 Heidelberg, Germany
}

Received 11 January 2006 / Accepted 10 February 2006

\section{ABSTRACT}

The young eruptive star V1647 Ori was observed with MIDI, the mid-infrared interferometric instrument at the Very Large Telescope Interferometer (VLTI), on March 2, 2005. We present the first spectrally resolved interferometric visibility points for this object. Our results show that (1) the mid-infrared emitting region is extended, having a size of $\approx 7 \mathrm{AU}$ at $10 \mu \mathrm{m}$; (2) no signatures of a close companion can be seen; (3) the $8-13 \mu \mathrm{m}$ spectrum exhibits no obvious spectral features. Comparison with similar observations of Herbig Ae stars suggests that V1647 Ori probably possesses a disk of moderate flaring. A simple disk model with $T \sim r^{-0.53}, \Sigma \sim r^{-1.5}, M_{\mathrm{d}}=0.05 M_{\odot}$ is able to fit both the spectral energy distribution and the observed visibility values simultaneously.

Key words. stars: formation - techniques: interferometric - stars: circumstellar matter - stars: individual: V1647 Ori - infrared: stars

\section{Introduction}

In January 2004 a new reflection nebula (McNeil's Nebula) appeared in the LDN 1640 dark cloud of the Orion B molecular cloud complex (McNeil et al. 2004). V1647 Ori, whose outburst ( $4 \mathrm{mag}$ in the $I$-band) caused the appearance of McNeil's Nebula, is a low-mass pre-main sequence object (Briceño et al. 2004; Ábrahám et al. 2004a). Its eruptive behaviour suggests that V1647 Ori is either an FU Orionis (FUor) or an EX Lupi (EXor) type object, or maybe an intermediatetype object between FUors and EXors (Muzerolle et al. 2005; Kóspál et al. 2005). Near-infrared colour maps show that the source is embedded in an elongated disk-like structure, whose size is approximately $7000 \mathrm{AU}$, and its inclination (the angle between the normal of the disk and the line of sight) is about $60^{\circ}$ (Acosta-Pulido et al., in prep.). The object had been gradually fading until October 2005, when the eruption rapidly ended (Kóspál et al. 2005).

Both FUors and EXors are low-mass pre-main sequence stars which exhibit optical brightening of several magnitudes. FUors are characterised by outbursts of several decades, while EXors exhibit repetitive outbursts on monthly timescale. The eruptive mechanism of both types is thought to be a rapid temporal increase of the disk accretion rate (Hartmann \& Kenyon 1996, and references therein). In this model the young star is accreting a substantial amount of material from the parent molecular cloud core $\left(10^{-6} M_{\odot} \mathrm{yr}^{-1}\right)$

* Based on observations made with the Very Large Telescope Interferometer at Paranal Observatory. via a circumstellar disk. The infalling matter piles up in the inner disk until its surface density - and thus opacity - becomes high enough to switch on a thermal instability leading to the dramatically increased accretion rate $\left(10^{-4}-10^{-3} M_{\odot} \mathrm{yr}^{-1}\right)$. It is still debated whether perturbation due to a close companion is needed to trigger the outburst, and it is also an open question whether all eruptive stars have companions (e.g. Wang et al. 2004; Reipurth \& Aspin 2004b).

The geometrical structure of the inner part of the circumstellar material in eruptive systems - though it is probably closely related to the outburst mechanism - is still unclear. Most models assume that apart from the circumstellar disk, there is an infalling remnant envelope of the molecular cloud core, whose inner radius is of the order of a few AU (Kenyon \& Hartmann 1991; Turner et al. 1997). This envelope supplies the disk with the material needed for the strong eruptions. In the case of V1647 Ori, Muzerolle et al. (2005) proposed that an optically thin envelope would be necessary to explain the flat infrared spectral energy distribution. However, a direct detection of such envelopes around eruptive stars has not been attempted so far.

In this paper we report on AU-scale observations of V1647 Ori at $10 \mu \mathrm{m}$ with MIDI, the mid-infrared interferometric instrument mounted at the Very Large Telescope Interferometer (VLTI) of ESO's Paranal Observatory (Leinert et al. 2003). We present spectrally resolved interferometric visibility points, and analyse the data with special emphasis on the structure of the circumstellar material and on the signature of a nearby companion. 


\section{Observations and data reduction}

V1647 Ori was succesfully observed with MIDI on the UT3UT4 baseline of the VLTI on March 2, 2005. The projected baseline length was $56 \mathrm{~m}$ with a $\mathrm{PA}=112^{\circ}$. Due to the lack of adequate guide star, $\mathrm{MACAO}^{1}$, could not support the observation, which was thus affected by the seeing $\left(0.9-1.0^{\prime \prime}\right.$ at the time of the observation). Single telescope N-band spectra were also taken with both UT3 and UT4. Additional N-band spectra were obtained on December 31, 2004 (then the interferometric part of the observation failed). On March 2, HD 37160 was observed as a calibrator, while on December 31 observations of HD 50778, performed 2 h later, were used.

The obtained data set consists of acquisition images with the N8.7 filter, $8-13 \mu \mathrm{m}$ low resolution spectra $(R=30)$, and interferometric measurements. In the data reduction we followed the general processing scheme as described by Leinert et al. (2004). The measured raw visibilities of V1647 Ori were divided by raw visibilities obtained from the HD 37160 data. The observations were reduced in two independent ways: with the MIDI Interactive Analysis (MIA) package, which uses the power spectrum method, and the Expert Work Station (EWS) package, which is based on a coherent, linear averaging method. For the extraction of the spectra the two packages ${ }^{2}$ consider fitted and fix masks, respectively. The interferometric results were then compared and showed agreement better than $10 \%$.

All available calibrator data obtained on March 2, 2005 were reduced in order to determine the errors of the spectrally resolved visibilities. After investigating the data we decided to apply a uniform multiplicative error of $10 \%$ as a conservative estimate.

At both epochs the N-band spectra obtained by the two telescopes differ in their absolute flux levels by a factor of $\approx 1.4$, and their spectral shapes slightly deviate at $\lambda>12 \mu \mathrm{m}$. The reason of this artifact is not yet clarified, thus we evaluated the acquisition images and extracted flux density values at $8.7 \mu \mathrm{m}$ for V1647 Ori (for the calibrator, HD 37160, 11.85 Jy was assumed). Photometry taken with UT3 turned out to be more reliable, judged from the repeatibility of the standard star measurements, and we estimate its absolute accuracy to be $15 \%$. The absolute calibration was then carried out by scaling the $\mathrm{N}$-band spectra to the photometric points at $8.7 \mu \mathrm{m}$.

\section{Results}

Figure 1 shows the calibrated visibilities as a function of wavelength. The values gradually decrease from 0.87 to 0.75 between 8 and $10 \mu \mathrm{m}$, and are approximately constant at longer wavelengths, with a small dip between 12 and $13 \mu \mathrm{m}$. This pattern clearly demonstrates that the emitting region is resolved, and suggests a non-uniform temperature distribution of the emitting material (otherwise the visibilities would monotonically increase towards longer wavelengths due to the lowering spatial resolution).

\footnotetext{
${ }^{1}$ http://www.eso.org/projects/aot/macao_vlti/, the Curvature AO system for the VLTI

${ }^{2}$ http://www.mpia-hd.mpg.de/MIDISOFT/
}

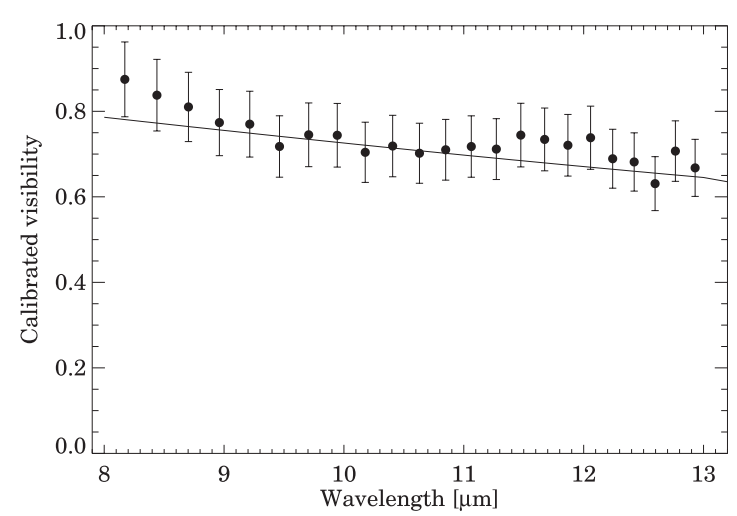

Fig. 1. Calibrated visibilities as a function of wavelength. The uniform error bars of $10 \%$ reflect our conservative estimate of the uncertainties. The solid line represents the model visibility curve of V1647 Ori (see Sect. 4).

Adopting Eq. (1) from Leinert et al. (2004), we calculated visibility values for a series of extended model sources of increasing sizes, represented by Gaussian brightness profiles, and compared them with our observations at different wavelengths. The derived FWHMs increase towards longer wavelengths from $6_{-3}^{+2}$ mas at $8.2 \mu \mathrm{m}$ to $16 \pm 3$ mas at $13 \mu \mathrm{m}$. At the distance of V1647 Ori (450 pc) these angular sizes approximately correspond to 2.7 and 7.2 AU linear sizes, respectively. The increasing sizes of Gaussians with increasing wavelength indicate a radially decreasing temperature profile.

Figure 2 presents our $8-13 \mu \mathrm{m}$ spectrum of V1647 Ori obtained with MIDI on UT3 in March 2005. The spectrum is smoothly rising towards longer wavelengths, and no obvious spectral features (silicates, PAHs, molecular ices) can be seen. The shape of the December 2004 MIDI spectrum is identical to the plotted spectrum within the measurement uncertainties. For comparison, in Fig. 2 we also overplotted an earlier spectrum of V1647 Ori from March 2004 (see figure caption). Comparison of the spectra suggests that the N-band spectrum changed dramatically (especially at longer wavelengths) between March and December 2004, but exhibited an approximately constant spectral shape afterwards, between December 2004 and March 2005.

We calculated the spectrum of the correlated flux as the product of the measured N-band spectrum and the spectrally resolved visibilities, in order to study the emission of the innermost part of the circumstellar structure. We found that the main fraction of the observed N-band flux $(\approx 70 \%)$ is emitted in the inner regions. The relatively flat shape of the correlated spectrum indicates that the innermost part has higher temperature. Like in the full spectrum, no spectral features can be observed in the innermost spectrum.

\section{Discussion}

V1647 Ori is a low-mass object (Briceño et al. 2004; Ábrahám et al. 2004a). Nevertheless, due to its increased outburst luminosity ( $L_{\mathrm{bol}}=44 L_{\odot}$, Muzerolle et al. 2005), it may look similar to intermediate-mass young stars. As the first step of our analysis, we compare our MIDI results 


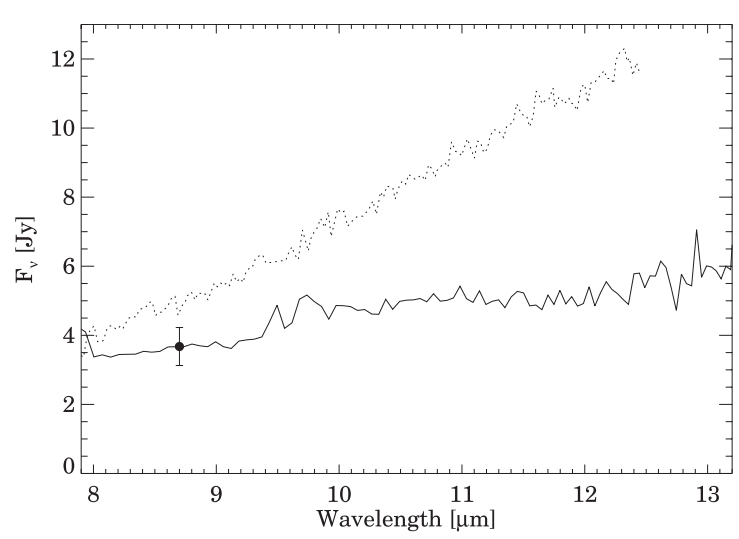

Fig. 2. N-band spectra of V1647 Ori at different epochs: solid line - VLTI/MIDI (March 2, 2005, this study); dotted line UKIRT/Michelle (March 11, 2004, Andrews et al. 2004). The MIDI spectrum was scaled to the photometric point at $8.7 \mu \mathrm{m}$ obtained with UT3 (filled dot). Neither the MIDI nor the UKIRT spectrum shows any obvious spectral features.

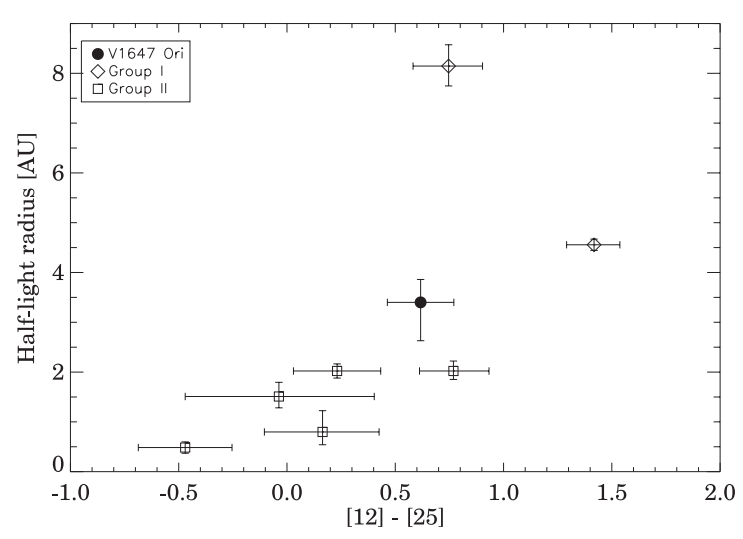

Fig. 3. Correlation between the mid-infrared spectral shape, calculated as [12]-[25] $=-2.5 \log \left(F_{v}(12 \mu \mathrm{m}) / F_{v}(25 \mu \mathrm{m})\right)$, and the half-light radius (adapted from Leinert et al. 2004, see text). V1647 Ori is marked with a filled circle.

with similar observations of a sample of Herbig Ae/Be stars (Leinert et al. 2004). Looking first at the visibility curves (Fig. 1 of the present paper; Fig. 4 in Leinert et al. 2004) one notices a general agreement between the shapes of the V1647 Ori and the Herbig Ae/Be curves: a faster drop in the $8-9 \mu \mathrm{m}$ range is followed by almost constant visibility values at longer wavelengths (exceptions are HD 179218 and $51 \mathrm{Oph}$ ). All Herbig Ae/Be stars show lower visibility values than V1647 Ori, indicating that these objects are more resolved. This finding can be explained to a large extent by distance differences: these Herbig Ae/Be stars are at a distance of 103-244 pc compared with the distance of V1647 Ori of $450 \mathrm{pc}$. We tested this hypothesis with the help of a disk model (see below) in which the distance was scaled from 450 to $150 \mathrm{pc}$, and found visibility curves similar to those of the Herbig Ae/Be stars.

In Fig. 3 we reproduced Fig. 5 of Leinert et al. (2004). In order to superpose V1647 Ori in the diagram, we computed the half-light radius as defined in Leinert et al. (2004), and estimated the [12]-[25] magnitude difference by interpolating among photometric points measured by the Spitzer

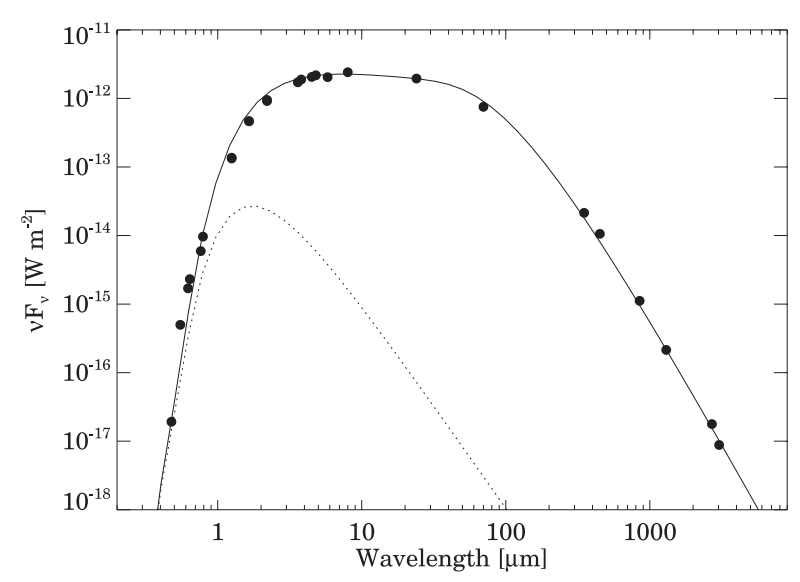

Fig. 4. Spectral energy distribution of V1647 Ori. All optical and infrared data plotted were obtained in February-March 2004. The thick line is the best fitting disk model (see text); dotted line represents contribution from the F0V star. Sources of data: VRI and $J H K$ : Acosta-Pulido et al., in prep.; $g^{\prime} r^{\prime} i^{\prime}$ and $J H K$ : Reipurth \& Aspin (2004a); 3.6-70 $\mu \mathrm{m}$ : Muzerolle et al. (2005); 3.8, 4.8 $\mu \mathrm{m}$ : Vacca et al. (2004); submm and mm-wavelength: Andrews et al. (2004), Lis et al. (1999), Mitchell et al. (2001), Tsukagoshi et al. (2005).

Space Telescope in March 2004 (Muzerolle et al. 2005). When plotting the Herbig Ae/Be stars, different symbols were used for Group I sources (thought to harbour flared disks, Meeus et al. 2001), and Group II sources (believed to possess non-flaring disks). V1647 Ori fits into the trend that redder disks are more extended. Considering its position between Group I and Group II sources one may conclude that V1647 Ori probably has a disk of moderate flaring. However, more complex models including additional components, such as envelopes, cannot be excluded.

FU Orionis, the prototype of the FUor class, was also observed with MIDI (Quanz et al. 2006), and - being located at the same distance - it is natural to compare FU Ori and V1647 Ori. The spectrally resolved visibility curves of FU Ori are very similar to that of V1647 Ori (at least for 2 of the 3 measured baselines) in both wavelength dependence and absolute value. Their N-band spectra are, however, somewhat different: FU Ori exhibit weak silicate emission, while V1647 Ori shows only smooth continuum radiation. Malbet et al. (2005) modelled FU Ori with a geometrically thin accretion disk which could reproduce their near-infrared interferometric results as well as the broad infrared-millimetre SED. The same model, however, failed to match the mid-infrared visibility curve obtained by MIDI (Quanz et al. 2006).

In order to model the spectrally resolved visibilities of V1647 Ori, first we constructed a spectral energy distribution. Fortunately, many observations were performed by different groups in February-March 2004, which cover the whole $0.5-850 \mu \mathrm{m}$ range and are now available in the literature. Though this date was one year earlier than our MIDI observation, V1647 Ori was in the outburst phase at both epochs. The SED is plotted in Fig. 4 (for references of individual observations see figure caption). The SED is characterised by a flat spectral shape ( $v F_{v} \approx$ const.) in the 3-30 $\mu$ m range, similarly to a number of FU Orionis objects, like V1057 Cyg, V346 Nor, 
Z CMa (Ábrahám et al. 2004b; Millan-Gabet et al. 2005). On the other hand, the SED of V1647 Ori significantly differs from that of FU Ori (Malbet et al. 2005).

We model the SED using a simple disk model. More complex configurations, e.g. flat disk plus envelope, might also be plausible, but the lack of any silicate emission in the N-band spectrum (Fig. 2) argues against the presence of an optically thin envelope (e.g. like the one proposed by Muzerolle et al. 2005). Our analysis tests the adequacy of simple disk models to simultaneously fit the SED and the MIDI visibilities. In our model one can specify the radial profiles of the temperature and the surface density in the disk, assuming that both follow a single power-law. We assumed surface density as $\Sigma \sim r^{-1.5}$, an extinction of $A_{\mathrm{V}}=10 \mathrm{mag}$, and an inclination angle of $60^{\circ}$ (Acosta-Pulido et al., in prep.). The central object was an F0V star, but its detailed properties did not affect considerably the fit. The fitted model parameters for the disk were the following: temperature $T(1 \mathrm{AU})=680 \mathrm{~K}$ and $T \sim r^{-0.53}$, inner and outer disk radii $7 R_{\odot}$ and $100 \mathrm{AU}$, respectively, disk mass $M_{\mathrm{d}}=0.05 M_{\odot}$. The best fit to the spectral energy distribution is overplotted in Fig. 4. The simple disk model matches all data from 0.5 to $3000 \mu \mathrm{m}$ with high precision.

We predicted visibilities from the above disk model according to the van Cittert-Zernicke theorem, i.e. the complex visibility is computed as the Fourier transform of the source brightness distribution, and vice versa. The resulting spectrally resolved visibility curve for the exact projected baseline of the V1647 Ori observation is overplotted in Fig. 1. The prediction agrees with the observed visibility points within the measurement uncertainties. Thus our simple disk model of V1647 Ori fits the spectral energy distribution and matches the visibility curve simultaneously. The model is also able to reproduce the slope of our $\mathrm{N}$-band spectrum. We note, that the canonical model usually proposed for FUors (an optically thick, geometrically thin accretion disk with $T \sim r^{-0.75}$ (Shakura \& Sunyaev 1973), or a flared disk with $T \sim r^{-0.75}$ in the inner disk and a shallower temperature profile in the outer regions) does not fit the data. However, our model with a shallower temperature profile of $T \sim r^{-0.53}$, whose exponent is similar to that of flared disk regions in standard models, can successfully reproduce the observations. Also, based on recent near-infrared interferometric measurements, Millan-Gabet et al. (2005) found that many FUors require a different model than the canonical one. An exception is FU Orionis itself, which was successfully modelled by Malbet et al. (2005) using a geometrically thin accretion disk.

Temporal evolution of the N-band spectrum of V1647 Ori (Fig. 2) may suggest a more complex picture of the circumstellar structure. The different fading rates at 8 and $13 \mu \mathrm{m}$ might indicate the presence of two physically separate components, which have different energy budget and cooling rate. Detailed time-dependent models will be necessary to describe simultaneouly the SED, the visibilities and the temporal evolution.

A companion would cause sinusoidal variations in the spectrally resolved visibilities with appropriate baseline position angles. The shape of our visibility curve suggests that no companion is present at the measured position angle whose separation is less than $100 \mathrm{AU}$ and brightness ratio is greater than $10 \%$. Nor do the acquisition images show any companion. The presence of companions is an important issue to discriminate between different hypotheses explaining the outburst mechanism (Reipurth \& Aspin 2004b). Due to the lack of more MIDI observations on baselines of different length and orientation, we can exclude the companion only along the measured baseline. The recent rapid fading of V1647 Ori (Kóspál et al. 2005) may prevent us from obtaining more visibility data in the present outburst period. A future brightening might offer the possibility to clarify the existence of a companion of brightness somewhat similar to the primary.

Acknowledgements. The work was supported by the grants OTKA T 034584 and K 62304 of the Hungarian Scientific Research Fund, and by the European Interferometry Initiative (Fizeau Programme). The authors thank Frank Przygodda for the help in the preparation of the MIDI observations, the ESO VLTI team (Garching/Paranal) for preparing and performing the service observations, and Attila Moór for his work on the absolute calibration of the MIDI spectra. The authors are also grateful to an anonymous referee, whose helpful comments improved the paper.

\section{References}

Ábrahám, P., Kóspál, Á., Csizmadia, Sz., et al. 2004a, A\&A, 419, L39 Ábrahám, P., Kóspál, Á., Csizmadia, Sz., et al. 2004b, A\&A, 428, 89 Acosta-Pulido, J., Kun, M., Ábrahám, P., et al., in prep.

Andrews, S. M., Rothberg, B., \& Simon, Th. 2004, ApJ, 610, L45

Briceño, C., Vivas, A. K., Hernández, J., et al. 2004, ApJ, 606, L123

Hartmann, L., \& Kenyon, S. J. 1996, ARA\&A, 34, 207

Kenyon, S. J., \& Hartmann, L. 1991, ApJ, 383, 664

Kóspál, Á., Ábrahám, P., Acosta-Pulido, J., et al. 2005, IBVS, 5661, 1 Leinert, Ch., Graser, U., Przygodda, F., et al. 2003, Ap\&SS, 286, 73

Leinert, Ch., van Boekel, R., Waters, L. B. F. M., et al. 2004, A\&A, 423, 537

Lis, D. C., Menten, K. M., \& Zylka, R. 1999, ApJ, 527, 856

Malbet, F., Lachaume, R., Berger, J.-P., et al. 2005, A\&A, 437, 627

McNeil, J. W., Reipurth, B., \& Meech, K. 2004, IAUC, 8284, 1

Meeus, G., Waters, L. B. F. M., Bouwman, J., et al. 2001, A\&A, 365, 476

Millan-Gabet, R., Monnier, J. D., \& Akeson, R. L. 2006, ApJ, in press [arXiv: astro-ph/0512230]

Mitchell, G. F., Johnstone, D., et al. 2001, ApJ, 556, 512

Muzerolle, J., Megeath, S. T., Flaherty, K. M., et al. 2005, ApJ, 620, L107

Quanz, S. P., Henning, Th., Leinert, Ch., et al. 2006, Proc. of The Power of Optical/IR Interferometry, in press

Reipurth, B., \& Aspin, C. 2004a, ApJ, 606, L119

Reipurth, B., \& Aspin, C. 2004b, ApJ, 608, L65

Shakura, N. I., \& Sunyaev, R. A. 1973, A\&A, 24, 337

Tsukagoshi, T., Kitamura, Y. Kawabe, R., et al. 2005, PASJ, 57, L21

Turner, N. J. J., Bodenheimer, P., \& Bell, K. R. 1997, ApJ, 480, 754

Vacca, W. D., Cushing, M. C., \& Simon, T. 2004, ApJ, 609, L29

Wang, H., Apai, D., Henning, Th., \& Pascucci, I. 2004, ApJ, 601, L83 